\title{
Validación de propuesta para medir la Interactividad en un ambiente de aprendizaje - caso plataforma Erudito
}

\author{
Ricardo Uribe', Néstor Duque-Mendez ${ }^{1}$, Julián Moreno² \\ ${ }^{1}$ Grupo de Investigación en Ambientes Inteligentes Adaptativos GAIA - Universidad \\ Nacional de Colombia - Sede Manizales \\ Km 9 vía al Aeropuerto La Nubia - Manizales-Colombia \\ ${ }^{2}$ Grupo de investigación en informática educativa - Universidad Nacional de Colombia \\ - Sede Medellín \\ \{riuribego, ndduqueme, jmoreno1\}@unal.edu.co
}

\begin{abstract}
One of the relevant factors in learning environments is interactivity, directly related to the activities and contents provided. Despite its importance, there is no universally accepted definition or measurement mechanism. This paper presents a conceptual model for quantitative measurement, based on specific metrics. The proposal was validated in a virtual learning environment, making the calculations with the data obtained from the educational platform.
\end{abstract}

Resumen. Uno de los factores relevantes en los ambientes de aprendizaje es la interactividad, directamente relacionada con las actividades y contenidos provistos. A pesar de su importancia, no existe una definición ni mecanismos para su medición aceptados universalmente. Este trabajo presenta un modelo conceptual para medición cuantitativa, basadas en métricas especificas. La propuesta fue validada en un ambiente de aprendizaje virtual, realizando los cálculos con los datos obtenidos desde la plataforma educativa.

\section{Introducción}

Los ambientes de aprendizaje buscan ofrecer las mejores características orientadas a mejorar el nivel de aprendizaje de los alumnos que los utilizan. Algunos se orientan a mejorar la usabilidad, la accesibilidad, la interacción, la amigabilidad, entre otros aspectos.

La interactividad a pesar de ser un factor relevante para el aprendizaje ha sido menos explorado y desarrollado tanto en lo conceptual como en lo practico en estos entornos. Algunos sistemas ofrecen a sus alumnos un nivel de interactividad elevado (Gutiérrez, 2011), pero soportados en lo expresado por el creador o administrador del ambiente, pero no se cuenta con la forma de medir la interactividad entre un ambiente y otro y poder comparar su nivel (Guevara, Botero, \& Castro, 2015).

En el caso concreto de la usabilidad se ha venido consolidando un soporte conceptual y mecanismos concretos para su evaluación. No obstante, en el caso de la interactividad aún se encuentran definiciones confusas o que se asocian más a la interacción o que recogen otros componentes de los ambientes educativos, por lo cual podemos decir que no existe una definición completa y universalmente aceptada. Producto de esta misma situación, existen variantes, pero no una y comúnmente adoptada forma de medir los diferentes componentes involucrados y así poder, comparativamente, conocer las diferencias entre una $\mathrm{y}$ otra plataforma $\mathrm{u}$ ambiente $\mathrm{y}$ determinar los puntos a mejorar 
VII Congresso Brasileiro de Informática na Educação (CBIE 2018)

Anais do XXIX Simpósio Brasileiro de Informática na Educação (SBIE 2018)

para obtener un valor de interactividad mejor. Pero, además, se requiere contar con herramientas tecnológicas que permitan la medición de la interactividad en la mayoría de los ambientes o que se disponga con los datos para realizar la medición objetiva (Liuber \& Álvarez, 2017), (Gil Pérez, Ana y Berlanga Fernández \& La, 2013),.

Este trabajo presenta un modelo conceptual para medición cuantitativa que recoge varios de los aspectos recogidos de la revisión de la literatura y que define una serie de métricas que integradas permiten conocer el nivel de interactividad de un escenario bajo evaluación. La propuesta fue validada en un ambiente de aprendizaje virtual llamado Erudito y los cálculos se realizaron con datos obtenidos, en gran medida, directamente desde la plataforma educativa.

\section{Propuesta conceptual y validación}

La Figura 1 presenta el modelo de evaluación de la interactividad en ambientes de aprendizaje propuesto, que recoge varios de los elementos del estado de arte.

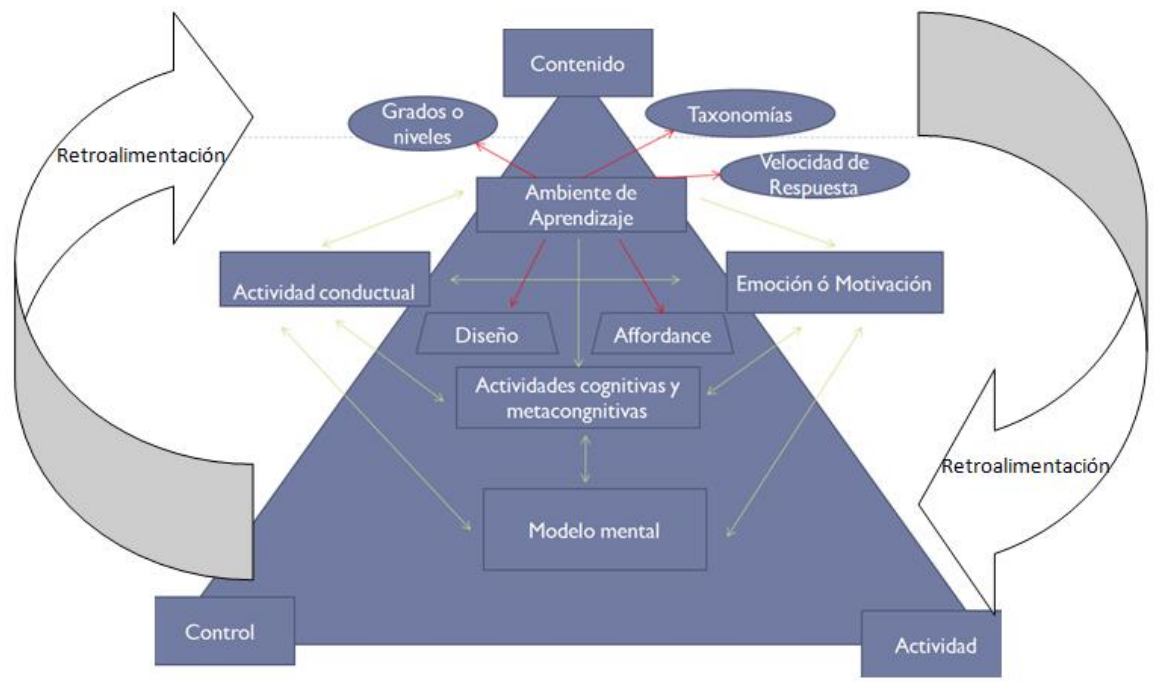

Figura 1. Modelo de la interactividad integral. Basado en los modelos de: (Zangara \& Sanz, 2013) y los conceptos de las características de la interactividad de (Greiner \& Ball, 1999)(Guevara et al., 2015)(Milojevic, Kleut, \& Ninkovic, 2013)(Veraszto, García, \& Silva, 2009).

Como se aprecia en la figura involucra los siguientes aspectos: Tiempo y velocidad de respuesta, retroalimentación total y parcial participación, el grado de interactividad, el affordance, el control vs libertad y nivel cognoscitivo de la acción. Cada uno de estos términos es calculado y el resultado es la evaluación de la interactividad en la plataforma. Las formulas detalladas son presentadas en (Uribe, Duque-Mendez y Moreno, 2018).

Para el proceso de validación del modelo, se diseñó un proceso basado en TIC para la medición de cada término de la propuesta. Se pudo calcular directamente a través de código en forma automática la participación, retroalimentación, el tiempo vs velocidad de respuesta, el grado de interactividad y los niveles cognitivos. Se aplicaron mecanismos para obtención de datos desde el interior de la plataforma Erudito, como el caso de la técnica scraping. 
VII Congresso Brasileiro de Informática na Educação (CBIE 2018)

Anais do XXIX Simpósio Brasileiro de Informática na Educação (SBIE 2018)

\section{Procesos para medición y resultados}

Para la medición se utilizó el método Scraping o raspado, el cual consiste en utilizar la estructura de la aplicación web, en su conjunto llamado DOM (Document Objet Model ó Modelo de Objeto de Documento). Esta extracción se realizó sobre algunas de las aplicaciones de Erudito, con apoyo del administrador del ambiente de aprendizaje. A continuación se muestran algunos de los resultados.

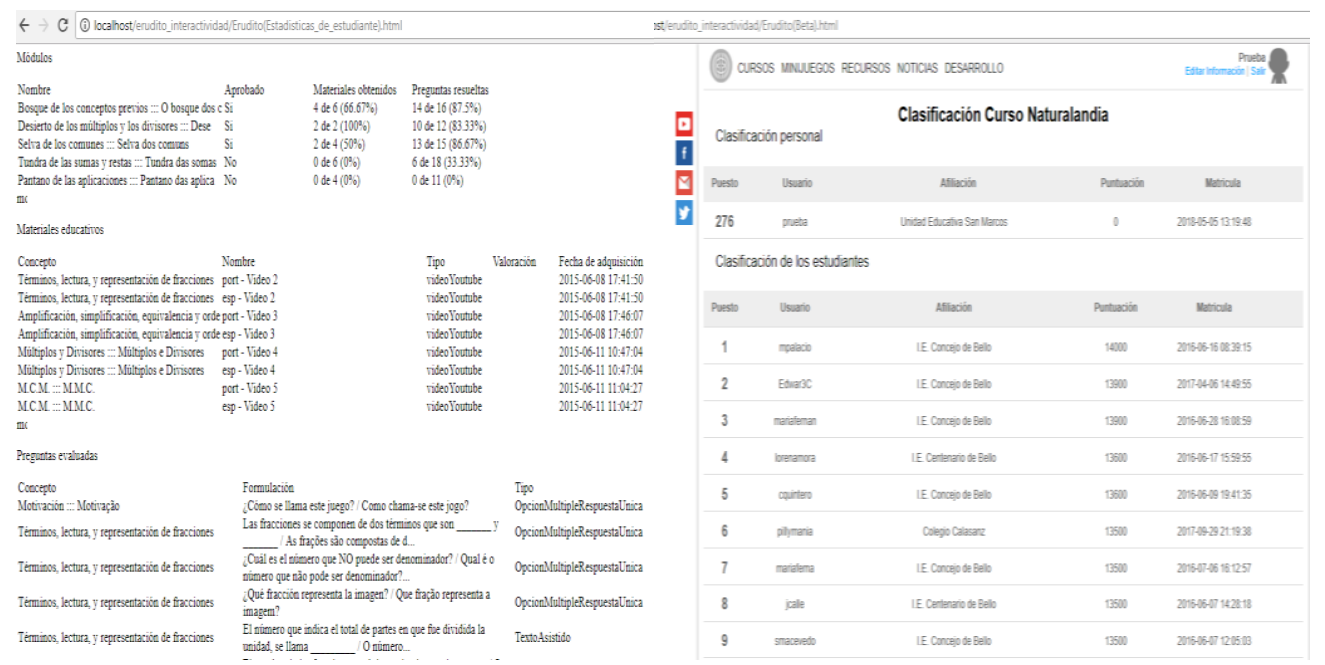

Figura 3. Aplicación del registro de un curso en el ambiente Erudito.

Con las aplicaciones anteriores se utiliza Scraping, desarrollado en lenguaje php y utilizando la función simple_html_dom de la librería del mismo nombre, la cual permite crear las aplicaciones con los datos que se pueden extraer o raspar del documento web o aplicación, lo cual favorece los cálculos propuestos. Se hace un reconocimiento de la estructura de la página en el DOM de la aplicación, y de allí se guarda el código en estructuras de almacenamiento como variables, arreglos, etc. como se muestra en la Figura 3.

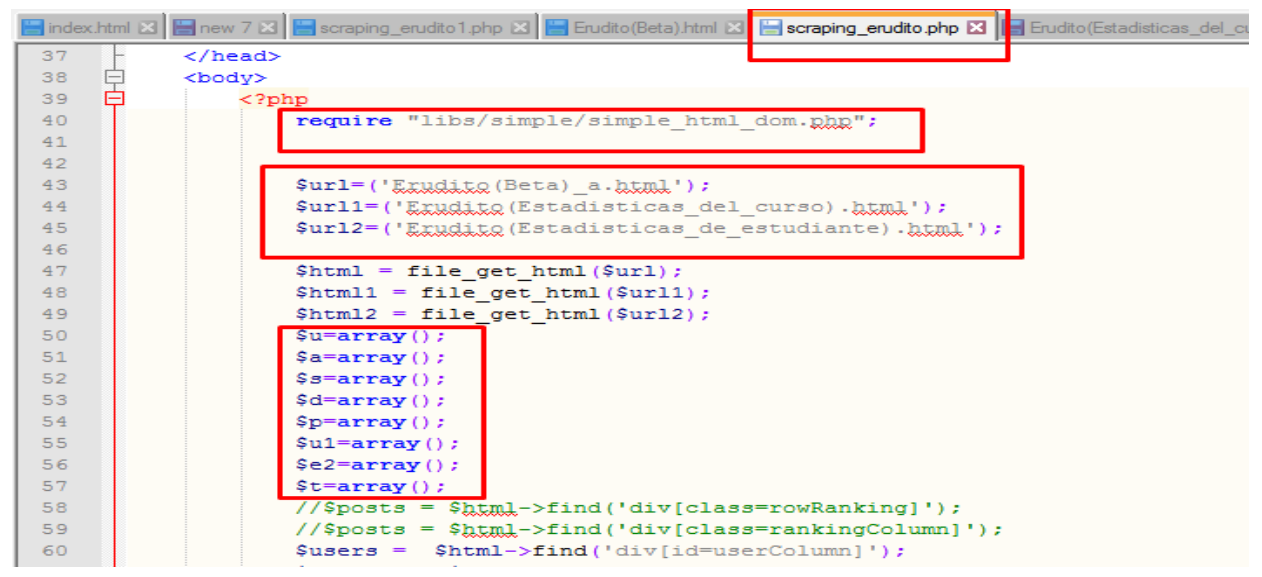

Figura 3. Muestra del código Scraping utilizado para el ambiente erudito.

El proceso de Scraping realizado arroja los resultados mostrados en la Figura 4. 
Medición de la Interactividad Erudito

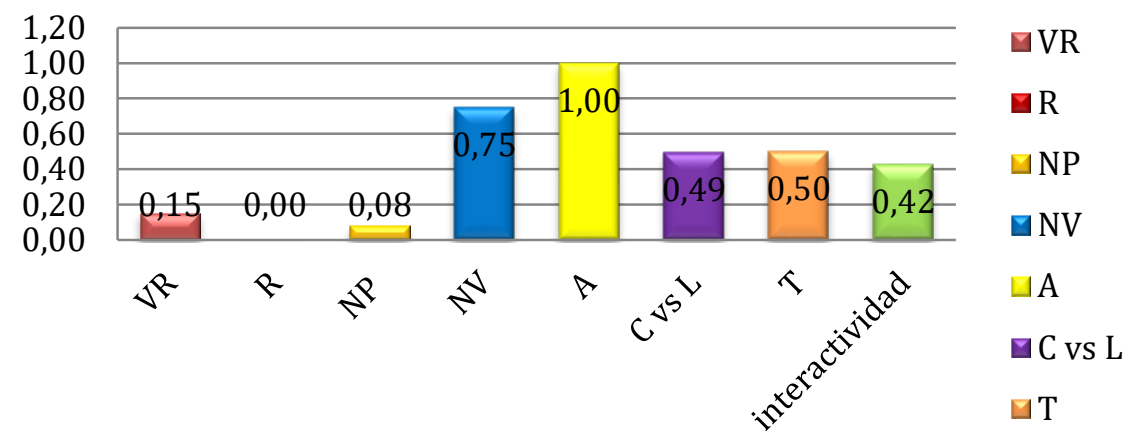

Figura 4. Grafica del resumen de la medición de la interactividad en el Erudito con todas sus características medibles.

Se aprecia que el ambiente Erudito obtuvo una velocidad de respuesta (VR) y Retroalimentación (R) muy baja, aclarando que solo se contó con la información de las acciones de uno de los alumnos lo cual no es concluyente. En el nivel de participación (NP), este arrojo un bajo resultado dado que no se pudo medir la participación de todos los alumnos. El grado de interactividad (NV) es complejo, dado a su capacidad necesarias para realizar este tipo de tareas. En el tema del affordance (A) se ve que el sistema tiene todo el potencial para que pueda ser altamente interactivo, eso dependerá del progreso y objetivos en sus actualizaciones, el control y libertad ( $\mathrm{C}$ vs $\mathrm{L}$ ), se ve un equilibrio lo cual es bueno para la interactividad, con opciones de progresar en el tema. Respecto al nivel cognoscitivo (T) está ubicado en la franja de las plataformas que apoyan la aplicación del conocimiento entregado lo cual lo ubica dentro del nivel 3 del modelo. Finalmente aplicando la fórmula propuesta el modelo arroja que el sistema Erudito tiene un nivel de interactividad media de $\mathbf{0 , 4 2}$, tal como se muestra en la figura 4.

\section{Conclusión y trabajo futuro.}

La propuesta presentada define una serie de componentes y métricas para el cálculo del nivel de interactividad de un ambiente de aprendizaje. Los cálculos se soportan en métricas concretas y que en gran medida puedes ser calculadas automáticamente desde los datos recogidos desde la plataforma misma. La propuesta es genérica, pues usando los mecanismos de extracción de datos es posible realizar los cálculos. La validación realizada confirma esta afirmación. Como trabajos futuros en marcha se amplía la evaluación en otros ambientes y consolidación de las métricas.

\section{Agradecimientos}

El trabajo presentado en este artículo se enmarca en el proyecto "Modelo orientado a la accesibilidad y adaptabilidad en Repositorios y Federaciones de Recursos Educativos Digitales" con código 35806 y financiado por la Universidad Nacional de Colombia. 
VII Congresso Brasileiro de Informática na Educação (CBIE 2018)

Anais do XXIX Simpósio Brasileiro de Informática na Educação (SBIE 2018)

\section{Referencias}

Gil Pérez, Ana y Berlanga Fernández, I., \& La. (2013). La interactividad en el aula. Un reto de la escuela 2.0, 2(2), 56-75.

Greiner, T. M., \& Ball, K. a. (1999). Understanding Interactivity. Computer Methods in Biomechanics and Biomedical Engineering, 12(2), 185-195. https://doi.org/10.1080/10255840802372060

Guevara, R., Botero, R., \& Castro, C. A. (2015). Una revisión a los niveles de interactividad de los contenidos digitales, 469-473.

Gutiérrez, M. C. (2011). LA INTERACTIVIDAD EN LA ENSEÑANZA Y EL APRENDIZAJE DE BÚSQUEDA DE INFORMACIÓN ELECTRÓNICA, EN UN AMBIENTE BIMODAL.

Lamas, C. (2013). Los medios interactivos y su publicidad. La medición de audiencias, $1-8$.

Liuber, M. S., \& Álvarez, G. (2017). CHARACTERIZATION OF THE EVALUATION OF INTERACTIVITY IN VIRTUAL ENVIRONMENTS OF TEACHING LEARNING AT THE UNIVERSITY OF HOLGUIN.

Milojevic, A., Kleut, J., \& Ninkovic, D. (2013). Methodological Approaches to Study of Interactivity in Communication Journals/Propuestas metodológicas para el estudio de la interactividad en revistas de comunicación. Comunicar, 21(41), 93-102.

Veraszto, E. V., García, F. G., \& Silva, D. (2009). LA EDUCACIÓN Y LA INTERACTIVIDAD Posibilidades inovadoras Sérgio Ferreira do Amaral Resumen Palabras clave Key words Introducción La aparición de una nueva tecnología puede ser, 655-665.

Uribe, R, Duque-Mendez, N., Moreno, J. Propuesta para la medición de la interactividad en ambientes de enseñanza-aprendizaje. revista Espacios. Venezuela. 2018

Zangara, A., \& Sanz, C. V. (2013). RUEDA - $6^{\circ}$ Seminario Internacional Mendoza Octubre 2013 Seminario Internacional Mendoza - Octubre 2013. Red de Universidades de Educación a Distancia Argentina, Seminario Internacional, 113. 